\section{Molecular antibiogram in diagnostic clinical microbiology: advantages and challenges}



\author{
“...molecular antibiogram cannot be \\ regarded as a standalone test, replacing \\ conventional antibiogram, but rather as a \\ supplemental test which can provide some \\ useful additional information. .."
}

Fabio Arena ${ }^{\ddagger 1}$, Tommaso Giani ${ }^{\ddagger 1}$, Simona Pollini ${ }^{2}$, Bruno Viaggi ${ }^{3}$, Patrizia Pecile ${ }^{4}$ \& Gian Maria Rossolini ${ }^{*, 2,4}$

First draft submitted: 8 February 2017; Accepted for publication: 8 February 2017; Published online: 31 March 2017

Antimicrobial susceptibility testing (AST) is a key activity in diagnostic microbiology. AST is based on testing the ability of antimicrobial agents to inhibit the growth of clinical isolates under standardized experimental conditions (antibiogram), which can be performed by various methods including broth microdilution, agar dilution, disk diffusion and gradient strip diffusion. The results of antibiogram are read as minimum inhibitory concentrations (MICs) or growth inhibition zone diameters around disks, and then translated into susceptibility categories according the clinical breakpoints defined by various Committees (e.g., CLSI, EUCAST). AST results are used to predict clinical efficacy of the tested antibiotics and to guide both definitive and empiric antimicrobial chemotherapy, when taken on an individual basis or on a cumulative basis representing the local epidemiology of antimicrobial susceptibility patterns, respectively [1].

The need for accurate AST data has increased in the current scenario of growing antibiotic resistance, where antimicrobial stewardship has become increasingly important to improve the clinical outcomes while minimizing the pressure for resistance selection [2].

Conventional antibiogram is based on phenotypic detection of bacterial growth, which requires a timeframe of 16-24 h with rapidly growing pathogens or even longer with slowly growing pathogens [1]. Recently, the possibility of detection of some resistance mechanisms at the molecular level in a significantly shorter timeframe has become available in diagnostic practice. This approach, indicated

\section{KEYWORDS}

- antimicrobial susceptibility testing

- diagnostic microbiology

- molecular detection 


\author{
"Rapidity of results, with a \\ time-to-response that can \\ be as short as $0.5-1 \mathrm{~h}$, is \\ one of the major \\ advantages of molecular \\ antibiogram. Moreover, \\ molecular antibiogram can \\ also work on positive \\ blood cultures or directly \\ on clinical specimens, \\ allowing a further \\ reduction of the time to \\ response.”
}

as molecular antibiogram (MA), was originally developed for slowly growing or fastidious pathogens (e.g., mycobacteria, Helicobacter pylori) $[3,4]$ but is now available also for several clinically relevant resistance mechanisms of common pathogens (e.g., staphylococci, enterococci, Enterobacteriaceae, and Gram-negative nonfermenters) and has become very attractive to deliver faster results on antimicrobial susceptibility, with a potentially remarkable impact on antimicrobial stewardship and clinical outcomes $[5,6]$.

However, several issues must be considered for implementation of MA in the diagnostic workflow. In this editorial we offer a perspective about those issues, by answering some questions encountered upon the implementation of MA in the diagnostic routine.

\section{Which technologies \& systems are available for MA?}

MA detects clinically relevant resistance mechanisms at the molecular level, with the primary aim of predicting clinical resistance to antimicrobial agents affected by those mechanisms. Detection usually targets resistance genes, using gene amplification technologies coupled with analysis of amplicons (e.g., real-time PCR, PCR plus microarrays, or PCR plus ESI-MS) [5], but can also target resistance proteins (e.g., a $\beta$-lactamase) by means of fast immune chromatography [7] or mass spectrometry [8], although the latter approach is still largely investigational. Being an area of intensive research, additional technologies and approaches for MA are expected for the near future.

The resistance mechanisms targeted by commercially available systems may differ in number and types [9]. This feature must be carefully considered when selecting a commercial diagnostic system for MA. To maximize the returned information, the system should be able to cover the most relevant resistance mechanisms encountered in the local epidemiology. For instance, if different types of carbapenemases are known to be present among strains of carbapenem-resistant Enterobacteriaceae spreading in the clinical setting, then systems for MA covering the different carbapenemase genes are preferable to those covering a single gene, even if it is the most prevalent.

\section{What are the major advantages of MA?}

Rapidity of results, with a time-to-response (TTR) that can be as short as $0.5-1 \mathrm{~h}$, is one of the major advantages of MA. Moreover, MA can also work on positive blood cultures or directly on clinical specimens, allowing a further reduction of the TTR. With some specimens (e.g., blood), however, the nucleic acid target amount is low and the extraction step may require more complex procedures and expensive systems, resulting in somewhat longer TTRs (5-6 h) [10].

Another advantage of MA is represented by the possibility to work also with polymicrobial specimens and to detect the molecular target, so in the presence of nonliving bacteria, due to previous exposure to antimicrobial agents, allowing an overall higher detection sensitivity when working directly with clinical specimens.

Indeed, some evidences supporting the benefits of MA in terms of reduction of length of stay in hospital and of healthcare-associated costs are already available $[6,11,12]$, although a recent Health Technology Assessment (HTA) analysis concluded that existing data regarding molecular techniques for pathogen identification directly from blood and MA are insufficient to demonstrate any improvement in key patients outcomes [13]. However, in that review, it is complicated to extrapolate the impact of the MA alone, since the information regarding detection of resistance genes is always considered together with pathogen identification.

\section{What are the major limitations of MA?}

Apart from the cost, which is usually higher than that of conventional antibiogram, a limitation of MA derives from the relatively narrow repertoire of detectable resistance mechanisms covered by the available systems, which implies a more limited information in comparison with conventional antibiogram. Indeed, results of MA are a useful proxy to predict resistance but less so to predict susceptibility (i.e., the lack of a certain resistance mechanism may not be sufficient to warrant susceptibility). Moreover, given its design, MA is intrinsically unable to detect unknown resistance mechanisms. These limitations could eventually be overcome when MA will be based on information covering the entire resistome of the clinical isolate. In fact, a good correlation between resistome analysis carried out by whole genome sequencing and conventional antibiogram has been reported with Staphylococcus aureus isolates $[14,15]$. However, the TTR of these whole genome sequencing-based methodologies is not yet compatible with the use in the diagnostic routine for clinical purposes. 
Another limitation is represented by the qualitative nature of MA results: no MICs are provided, while MIC values are increasingly relied upon for antimicrobial stewardship, especially with some drugs and pathogens [16].

Finally, with MA, there is a risk of overcalling resistance due to the presence of targeted resistance genes that are either inactivated or not expressed.

Due to these limitations, MA cannot be regarded as a standalone test, replacing conventional antibiogram, but rather as a supplemental test which can provide some useful additional information, crucial to antimicrobial stewardship and/or infection control policies, in a shorter timeframe.

\section{How to implement MA in the diagnostic workflow?}

As an expensive add-on to the diagnostic workflow, which cannot replace conventional antibiogram, MA represents an additional cost for the diagnostic laboratory in terms of reagents, equipment and manpower. Introduction of MA may also require a revision of the diagnostic workflows, to maximize its advantages. In fact, the overall impact of MA is expected to be influenced by the opening hours of the laboratory, being higher if the laboratory is operating on a $24 \times 7$ regimen.

Under these circumstances, MA cannot be used with all specimens and must be positioned within selected diagnostic pathways, following the principles of diagnostic stewardship. In our setting, for instance, MA has been incorporated in the diagnostic workup of positive blood cultures from high-risk patients from some hospital wards (Intensive Care Units, Oncohematology Unit and Stem Cell Transplantation Unit), prioritized according to a multiparametric score that takes into account clinical scores (e.g., SOFA score, PITT score, modified CPIS score and MDR score) and two biomarkers including procalcitonin and proadrenomedullin $[17,18]$.

\section{How to report results of MA?}

MA primarily provides information on the probability of resistance to some antibiotics, including those affected by the detected resistance mechanism. MA can also provide information on potential susceptibility to some antibiotics among those escaping the detected resistance mechanisms. The latter information, however, may be strongly influenced by the coverage of resistance mechanisms, the bacterial species and the local epidemiology of resistance.

As such, the information returned by MA is remarkably different from that of conventional antibiogram. On the other hand, clinicians are usually not familiar with this type of information and its significance. Therefore, when introducing MA into the diagnostic laboratory, a major challenge is represented by reporting results of MA to clinical users.

In our experience, results of MA are reported as the presence or absence of a resistance mechanism out of a list of the searched mechanisms, accompanied by a comment which informs on the likelihood of resistance to certain antibiotics or antibiotic classes, depending on the resistance mechanism that has been detected and on the bacterial species that has been identified in the clinical specimen. Information on potential susceptibility is also added whenever possible, especially for antibiotics which are known to be active against most isolates of some species and affected by specific resistance mechanisms (e.g., glycopeptides for enterococci, ceftolozanetazobactam for Pseudomonas aeruginosa and ceftazidime-avibactam for Enterobacteriaceae).

\section{Conclusion}

MA offers a unique opportunity to provide additional information on antimicrobial susceptibility of clinical isolates within a reduced timeframe, with a potentially significant impact on antimicrobial stewardship. Exploitation of this potential largely depends on the awareness of advantages and limitations of currently available systems for MA, on the selection of the most suitable MA systems for the operational setting, and on the adequate positioning of MA in the diagnostic workflow of the laboratory. The latter should be based on discussion and agreement with clinical users, who should become knowledgeable of these new diagnostic technologies.

Financial \& competing interests disclosure

$F$ Arena has received congress lecture fees from Accelerate Diagnostics, Alifax, bioMérieux and Biotest. T Giani has received a research grant from AstraZeneca and congress lecture fees from Alifax, bioMérieux, Thermo Fisher Scientific and Accelerate Diagnostics. B Viaggi has received congress lecture fees from Abbott, Accelerate Diagnostics, Alifax, Bellco, Merck, Pfizer and Thermo Fisher Scientific. P Pecile has received congress lecture fees from bioMérieux. GM Rossolini has received research grants from Accelerate Diagnostics, Alifax, Angelini
"Molecular antibiogram cannot be used with all specimens and must be positioned within selected diagnostic pathways, following the principles of diagnostic stewardship.” 
ACRAF, AstraZeneca, Basilea, Becton-Dickinson, bioMérieux, Biotest, Cepheid, Check-Points, ELITech, Estor, Liofilchem, Merck, Novartis, Nordic Pharma, Pfizer, Rempex/The Medicine Company, VenatoRx, Zambon; has received congress lecture fees from Angelini ACRAF, AstraZeneca, Basilea, Biotest, Merck, Pfizer; has received consultancy fees from Achaogen, Angelini ACRAF, AstraZeneca, Curetis, ELITech, Menarini, Merck, Nordic
Pharma, Pfizer, Rempex/The Medicine Company, Zambon. The authors have no other relevant affliations or financial involvement with any organization or entity with a financial interest in or financial conflict with the subject matter or materials discussed in the manuscript apart from those disclosed.

No writing assistance was utilized in the production of this manuscript.

\section{References}

Papers of special interest have been highlighted as: - of interest; $\bullet \bullet$ of considerable interest

1 Courvalin P, Leclercq R, Rice LB. Antibiogram (3rd Edition). ASM Press, Washington, DC, USA (2010).

2 Barlam TF, Cosgrove SE, Abbo LM et al. Implementing an antibiotic stewardship program: guidelines by the Infectious Diseases Society of America and the Society for Healthcare Epidemiology of America. Clin. Infect. Dis. 62(10), e51-e77 (2016).

3 Gazi MA, Islam MR, Kibria MG, Mahmud Z. General and advanced diagnostic tools to detect Mycobacterium tuberculosis and their drug susceptibility: a review. Eur. J. Clin. Microbiol. Infect. Dis. 34(5), 851-861 (2015).

4 Mégraud F, Bénéjat L, Ontsira Ngoyi EN, Lehours P. Molecular approaches to identify Helicobacter pylori antimicrobial resistance. Gastroenterol. Clin. North Am. 44(3), 577-596 (2015).

5 Van Belkum A, Dunne WM Jr. Nextgeneration antimicrobial susceptibility testing. J. Clin. Microbiol. 51(7), 2018-2024 (2013).

- A comprehensive overview of innovative methods for antimicrobial susceptibility testing.

6 Timbrook TT, Morton JB, McConeghy KW, Caffrey AR, Mylonakis E, LaPlante KL. The effect of molecular rapid diagnostic testing on clinical outcomes in bloodstream infections: a systematic review and meta-analysis. Clin. Infect. Dis. 64(1), 15-23 (2017).

- An extensive evaluation on the impact of molecular rapid diagnostic testing on clinical outcome in bloodstream infections.
7 Glupczynski Y, Evrard S, Ote I et al. Evaluation of two new commercial immunochromatographic assays for the rapid detection of OXA- 48 and KPC carbapenemases from cultured bacteria. J. Antimicrob. Chemother. 71(5), 1217-1222 (2016).

8 Van Belkum A, Chatellier S, Girard V, Pincus D, Deol P, Dunne WM Jr. Progress in proteomics for clinical microbiology: MALDI-TOF MS for microbial species identification and more. Expert Rev. Proteomics 12(6), 595-605 (2015).

9 Arena F, Viaggi B, Galli L, Rossolini GM. Antibiotic susceptibility testing: present and future. Pediatr. Infect. Dis. J. 34(10), 1128-1130 (2015).

10 Opota O, Jaton K, Greub G. Microbial diagnosis of bloodstream infection: towards molecular diagnosis directly from blood. Clin. Microbiol. Infect. 21(4), 323-331 (2015).

- An update on recent developments on the direct molecular diagnosis of bloodstream infections.

11 Bauer KA, West JE, Balada-Llasat J-M, Pancholi P, Stevenson KB, Goff DA. An antimicrobial stewardship program's impact with rapid polymerase chain reaction methicillin-resistant Staphylococcus aureus/S. aureus blood culture test in patients with S. aureus bacteremia. Clin. Infect. Dis. 51(9), 1074-1080 (2010).

- A clinical study assessing the impact of molecular antibiogram in patients with bloodstream infections.

12 Sango A, McCarter YS, Johnson D, Ferreira J, Guzman N, Jankowski CA. Stewardship approach for optimizing antimicrobial therapy through use of a rapid microarray assay on blood cultures positive for Enterococcus species. J. Clin. Microbiol. 51(12), 4008-4011 (2013).

13 Stevenson M, Pandor A, Martyn-St James M et al. Sepsis: the LightCycler SeptiFast Test MGRADE $^{\circledR}$, SepsiTest ${ }^{\mathrm{TM}}$ and IRIDICA BAC BSI assay for rapidly identifying bloodstream bacteria and fungi - a systematic review and economic evaluation. Health Technol. Assess. 20 (46), 1-246 (2016).

14 Gordon NC, Price JR, Cole K et al. Prediction of Staphylococcus aureus antimicrobial resistance by whole-genome sequencing. J. Clin. Microbiol. 52(4), 1182-1191 (2014).

- A demonstration of whole-genome sequencing as a promising alternative to standard antimicrobial susceptibility testing for the prediction of antibiotic resistance.

15 Aanensen DM, Feil EJ, Holden MT et al. Whole-genome sequencing for routine pathogen surveillance in public health: a population snapshot of invasive Staphylococcus aureus in Europe. MBio 7(3), doi:10.1128/ mBio.00444-16 (2016).

16 Heil EL, Johnson JK. Impact of CLSI breakpoint changes on microbiology laboratories and antimicrobial stewardship programs. J. Clin. Microbiol. 54(4), 840-844 (2016).

17 Bassetti M, Carnelutti A, Peghin M. Patient specific risk stratification for antimicrobial resistance and possible treatment strategies in Gram-negative bacterial infections. Expert Rev. Anti-Infect. Ther. 15(1), 55-65 (2017).

18 Valenzuela-Sanches F, Valenzuela-Méndez B, Rodríguez-Gutiérrez JF. New role of biomarkers: mid-regional proadrenomedullin, the biomarker of organ failure. Ann. Transl. Med. 4(17), 329 (2016). 